\title{
Russie: Violences familiales et Torture
}

\author{
Le Groupe des Professionnels de la Santé d'Amnesty International, J. Molleyres
}

Amnesty International a lancé il y a une année une campagne afin de soutenir la lutte pour les droits humains sur tout le territoire de la Fédération de Russie.

Les graves violations des droits humains en Tchétchénie sont évidemment l'objet de la campagne d'Amnesty International, mais le présent appel traite d'autres violations des droits humains, comme la torture et les violences familiales qui sont constatées sur l'ensemble du territoire de la Fédération de Russie.

Bien que la Fédération de Russie ait ratifié de nombreux traités internationaux relatifs aux droits humains et ait mentionné dans sa Constitution que le droit international prévalait sur le droit national, les tribunaux russes s'appuient encore et toujours sur le Code pénal national, qui, jusqu'à ce jour, reste très lacunaire en ce qui concerne la torture. Le nouveau Code de procédure pénal, approuvé par la Douma en décembre 2001, ne comble pas toutes les lacunes du précédent Code.

En fait, des violations graves des droits humains continuent à être commises sans entraîner de sanctions. Elles sont souvent perpétrées par des responsables de l'application des lois et des membres des forces de sécurité russes, qui jouissent à ce sujet d'une véritable impunité.

Le but de la campagne d'Amnesty International est donc de responsabiliser les autorités russes dans les cas de violations des droits humains et de combattre l'impunité de ceux qui les commettent.

Dans cette lutte contre les violations des droits humains, le rôle des professionnels de la santé est primordial aussi bien dans les cas de violence familiale (surtout envers les femmes) que dans les cas de tortures ou de traitements cruels, dégradants et inhumains commis par des agents de l'état.

En Russie, il est très difficile d'obtenir les preuves médicales de torture pour de nombreuses raisons.

Ainsi, la victime d'un acte de violence ne peut demander aux autorités judiciaires d'être examinée médicalement. Cette demande doit passer par l'enquêteur, même si celui-ci est personnellement impliqué dans les traitements cruels, inhumains et dégradants ou les tortures. En outre, la présence de cet enquêteur est autorisée lors de l'examen médical de la victime, qui est ainsi privée de toute confidentialité. Enfin, cet examen ne peut être pratiqué que par un médecin fonctionnaire d'état, les examens pratiqués par un médecin indépendant n'ayant aucune valeur en justice.

Ces dispositions sont contraires aux standards internationaux d'éthique médicale et peuvent à l'évidence influencer l'examen médical et le rapport qui en est fait.

En ce qui concerne la violence familiale, celle-ci n'est pas reconnue comme un crime ou un délit et la loi ne permet même pas de sanctionner les auteurs de violence, par des mesures administratives ou des amendes. En 2002, les autorités russes ont elles-mêmes reconnu que 14000 femmes mouraient chaque année des suites de violences commises par leur conjoint ou leurs proches parents! Nombre de parlementaires russes désirent toutefois introduire cette question dans la législation.

Amnesty International recommande que:

- toutes les victimes de violence familiale et tous les détenus se plaignant de tortures soient examinés par des professionnels de santé qualifiés et indépendants et que cet examen soit fait en privé;

- l'autorité judiciaire accepte les rapports médicaux faits par des professionnels de santé qualifiés et indépendants;

- le Ministère de la Santé et la Société Médicale Russe donnent des lignes directrices claires et une formation adéquate aux médecins et au personnel de santé concernant l'examen médical et la rédaction de rapports, tels qu'ils sont codifiés dans le Protocole d'Istanbul, afin d'aider les victimes de torture ou de violence familiale.

En tant que médecins et membres d'Amnesty International, nous souhaitons vivement que les médecins suisses prennent part à cette campagne en faisant savoir à la Société Médicale Russe ainsi qu'aux autorités russes, en particulier aux Ministres de la Santé et de la Justice de ce pays, qu'ils soutiennent les recommandations citées ci-dessus et déplorent que la Russie n'ait pas encore mis en œuvre les dispositions figurant dans les principaux accords internationaux relatifs aux droits humains signés par elle entre les années 1960 et 1991.

N.B. Des modèles de lettres et une liste d'adresses en Russie sont à disposition de ceux qui le désirent à l'adresse de correspondance. 